\section{Photoreceptors for the beginner}

\section{Aubrey Knowles}

Photoreceptors: Their Role in Vision.

By Alan Fein and Ete Z. Szuts.

Cambridge University Press: 1983.

Pp.212. Hbk f17.50, \$34.50;

pbk £7.95, \$13.95.

OUR knowledge of retinal photoreceptors has increased considerably over the past $\mathbf{2 0}$ years, and our present ideas about their function have come from a variety of disciplines ranging from anatomy through chemistry to physics. This book meets a need by providing a comprehensive review at a fairly elementary level. The fact that it appears in the IUPAB Biophysics Series should not put off intending readers: the volume could equally well have been included in a series on zoology or chemistry.

Vision is a sense common to most animals, and it is now clear that the photoreceptors of all species have structural similarities and their visual pigment molecules are closely related. However a gulf exists between workers on the vision of vertebrates and those dealing with invertebrates, due to the different visual characteristics observed and to the use of different techniques. The authors have made a good job of pulling together a wealth of information from these two fields, but this has of necessity involved the critical selection of material.

My principal criticism of the book is of bias in this selection that arises from Drs Fein and Szuts's interest in invertebrate

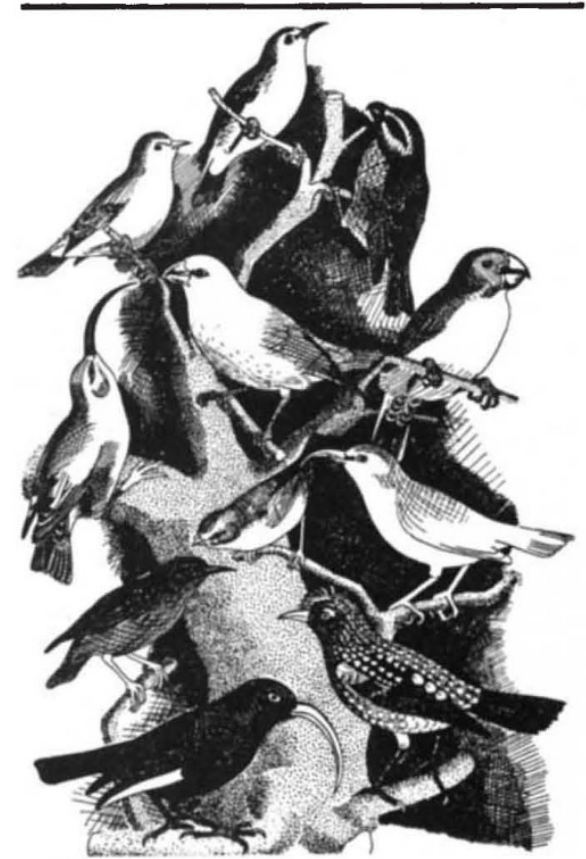

Adaptive radiation as displayed by Hawaiian sicklebills. The illustration is taken from Darwin's Finches by David Lack, recently reissued by Cambridge University Press with notes by Laurene Ratcliffe and Peter Boag. Price is hbk £19.50, \$39.50; pbk £7.95, \$13.95. vision. While this may merely reflect my interest in vertebrate photoreceptors, most students take an anthropomorphic view of vision, and can best tackle the subject as an extension of their own experience. The authors recognize this in the introductory chapters, where human visual performance is stressed, but this approach is abandoned as the text develops. In Chapter 8, which covers adaptation, vertebrates are barely mentioned. Cone receptors, colour vision and the visual performance of vertebrates receives poor coverage, although this area is of general interest and is the subject of active current research. The book thus attempts a useful service in bringing together the very different visual characteristics of insects and man, but fails to sustain this approach through to the end.

In another way the balance of the book is also not quite right, the early chapters being cluttered with detail while the later chapters become shorter and more cursory. Much of the early detail is irrelevant and

\section{The microfilament system}

\section{Uno Lindberg}

\section{Mechanisms of Cell Motility: \\ Molecular Aspects of Contractility. By Peter Sheterline.}

Academic: 1983. Pp.180. £14, \$23.

RESEARCH on the structural organization of eukaryotic cells in general and cell motility in particular was revolutionized by the development of antibodies against actin by Lazarides and Weber in 1974. Discovery of these antibodies led to a startling new picture of the distribution of actin in different kinds of cells and made it clear that chemo-mechanical transduction by the actomyosin system might be a fundamental process common to all eukaryotic cells. Exciting findings followed rapidly,and within a few years it became evident that eukaryotic cells have a complex and dynamically changing cytoskeleton consisting of finely tuned filament systems (microfilaments, microtubules, and intermediate filaments) through which the life processes can be directed and performed in a precise way. Peter Sheterline's book deals primarily with the microfilament system.

The molecular mechanisms underlying cell motility are still largely unknown. Many proteins which interact with the microfilament system have now been isolated, but the observations concerning their activity in vitro give only vague answers to several of the outstanding problems - how microfilaments are formed; how filopodia, microvilli and stress fibres are built by packing of actin filaments in paracrystalline bundles; how membrane lamellae extend themselves some is contentious - for example, the conjectured sensitizing pigment in fly receptors on p. 78 - while recent important work on single cell electrophysiology and the molecular mechanisms of transduction and adaptation are all compressed into the final 30 pages. The references are sensibly arranged at the end of the book and form a useful reading list. Unfortunately this list omits some useful recent reviews and contains a number of outdated and trivial references which will not be helpful to a newcomer to the field.

In summary, the book is a useful addition to the literature of vision, and will be helpful to students and research workers entering the field; I will recommend it to our second-year students in future. It is nicely produced though some of the figures are poor.

Aubrey Knowles is a Research Fellow in the Department of Biochemistry, University of Bristol.

from the surface of cells and might cause the observed translocations; and how all of these phenomena are regulated.

When lecturing on these matters I usually begin by describing the diversity of motile activity expressed by eukaryotic cells but emphasize that there might be a common basic mechanism. But to consider this it is necessary to have a relatively detailed picture of the organization of the individual structural elements of different types of cells and the different filament systems. After covering that I explain the difficulties involved in correlating the molecular organization of the cell structure with the behaviour of cells. Only then do I tackle the biochemistry of the components of the filament systems. I feel it is difficult for the beginner to see the molecular details but not lose track of the overall structural organization and behaviour of the cell.

Peter Sheterline has organized his book the other way around. He starts with a thoughtful discussion of the proteins involved in the microfilament system. Then he deals with the ultrastructural organization and with possible mechanisms. One could approach the book by taking a good look at the pictures of cells provided in Chapter 6 , and then read Chapters $1-5$ to find out more about the proteins involved. Then one might study carefully the concept of "contractile networks", but without taking the different models presented too seriously - they may be far from the reality of the living cell. Unfortunately, the author makes no attempt to tell how the story has developed, and he makes few references to original observations. So while there is something in the book to exercise the specialist, the newcomer should look elsewhere for an introduction to this field.

Uno Lindberg is a Professor at the WennerGren Institute, University of Stockholm. 